\title{
tDCS peripheral nerve stimulation: a neglected mode of action?
}

\section{The presumed mechanism of tDCS reconsidered}

Transcranial direct current stimulation (tDCS) is a non-invasive neuromodulation method in which a weak DC current is passed through at least two scalp electrodes. Neuroscientists use tDCS as a tool to study the functional roles of different brain regions, and investigate their behavioral and cognitive correlates. In a clinical context, tDCS is being investigated as treatment for a wide range of conditions, including addiction [1], depression [2], and cognitive decline [3]. Due to this broad potential, combined with its noninvasive nature and low-cost, tDCS is quickly growing in popularity, illustrated by its exponentially growing output of publications [4] and the growing number of consumer tDCS devices on the market.

While increasing amounts of research resources are directed towards tDCS's applications, remarkably little is known about the technique's underlying neurophysiological mechanisms. It is widely assumed that effects of tDCS are solely caused by the electric field that it generates in the brain. This weak field is believed to modify functioning of the brain by polarizing the membrane potential of neurons, and thereby altering their excitability [5]. In this opinion piece we would like to suggest that this may be an oversimplified representation of the mechanisms mediating tDCS's effects. In the following paragraphs we discuss evidence which supports this opinion.

There are currently three main non-invasive brain stimulation (NIBS) techniques in the neuroscience toolbox: tDCS, transcranial magnetic stimulation (TMS) [6], and transcranial alternating current stimulation (tACS) [7]. TMS uses a changing magnetic field to create an electric field in the desired brain area. It was generally assumed that this electric field stimulates cortical neurons which then cause all the observed TMS effects. However, recent research has shown that some TMS effects are not caused by direct stimulation of cortical neurons, but instead by off-target excitation of peripheral nerves in the scalp [8] [9]. Similarly, our group has recently demonstrated that tACS motor system effects can be caused by stimulation of peripheral nerves in the scalp [10]. Interestingly, tACS is highly similar to tDCS and differs only in its use of alternating current instead of direct current.

Somewhat surprisingly, the involvement of peripheral mechanisms has never been systemically investigated for tDCS, and appears to have been overlooked by the field (see recent review of tDCS mechanisms [11]). Meanwhile, interest in tDCS from both academia and the general public continues to grow. Consequently, it is now timely to ask the question 'Is there a role for peripheral nerve stimulation in mediating tDCS effects?'

\section{How does tDCS work?}

The neurophysiological mechanism (or more likely range of mechanisms) underpinning observed tDCS effects is currently poorly understood. tDCS electrodes generally apply currents between 1 and $2 \mathrm{~mA}$. To reach the cerebral cortex, these currents pass through the scalp, skull and cerebrospinal fluid. Consequently, the electric field is attenuated to less than $0.5 \mathrm{~V} / \mathrm{m}$ when it reaches the cortices just under the electrodes [12] [13]. While this field is too weak to initiate action potentials in cortical neurons, it can polarize their membrane potential [5]. We term this the tDCS transcranial mechanism (Figure 1). Importantly, tDCS could also exert its effects through a peripheral route. As tDCS electrodes are placed directly on the skin, peripheral nerves are exposed to higher electric field strengths that can reach $20 \mathrm{~V} / \mathrm{m}$ [14] [10]. This peripheral field is strong enough to initiate action potentials in peripheral 
nerves [15]. Consequently, stimulation of peripheral nerves activates the somatosensory system (causing the reported tingling sensation in tDCS [16]) as well as a plethora of other brainstem and limbic structures such as the locus coeruleus, amygdala and hippocampus [17] [18]. We term this the tDCS transcutaneous mechanism (Figure 1). The possible contribution of this mechanism to the observed tDCS effects has been mostly overlooked, as the standard tDCS sham condition does not control for the transcutaneous route.

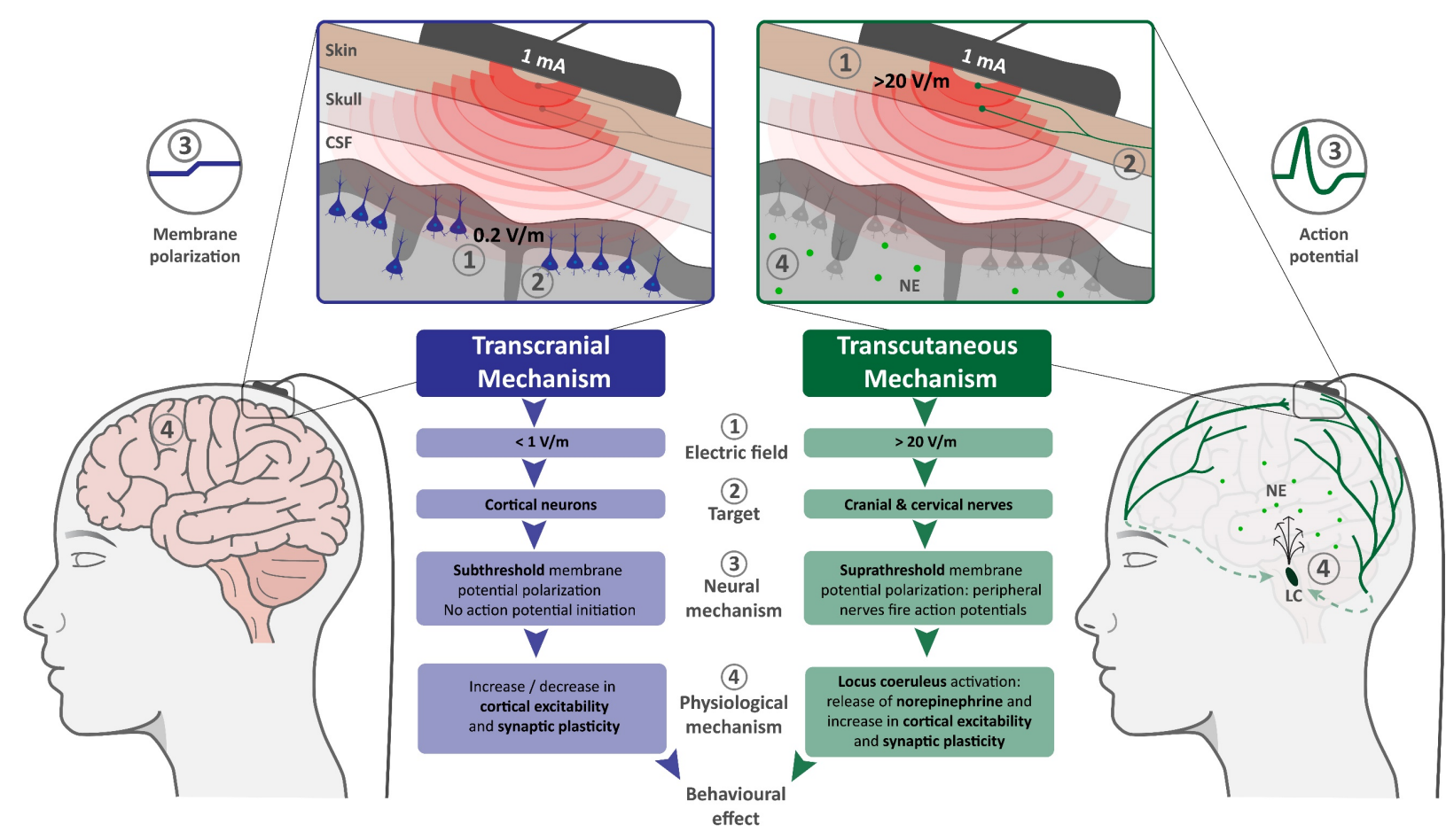

Figure 1. Comparison of the tDCS transcranial and transcutaneous mechanisms (CSF = cerebrospinal fluid, LC = Locus coeruleus, $\mathrm{NE}=$ Norepinephrine) .

\section{tDCS transcranial mechanism}

The typical cortical electric field strength generated when tDCS is applied is around $0.2 \mathrm{~V} / \mathrm{m}$ [12] [13]. As this is too low to directly initiate action potentials in neurons, it is proposed that the tDCS transcranial mechanism acts by polarizing the membrane potential. Work in brain slices shows that excitatory cortical neurons have an average polarization length constant of 0.2 [19], i.e. a $0.2 \mathrm{mV}$ membrane potential shift for an extracellular electric field strength of $1 \mathrm{~V} / \mathrm{m}$. When tDCS generates its typical electric field strength of $0.2 \mathrm{~V} / \mathrm{m}$, the membrane potential of excitatory neurons would thus shift by $0.04 \mathrm{mV}$. How could this small shift account for the significant changes in cortical excitability reported in human tDCS experiments [20]? Slice experiments provide a possible answer: it was recently shown that the shift in membrane potential at the synapse can be 4 times larger than the shift at the soma [21]. The typical shift of $0.04 \mathrm{mV}$ in an excitatory neuron during tDCS may thus cause a membrane potential shift of $0.16 \mathrm{mV}$ at its synaptic boutons. Interestingly, the study showed that this polarization of axon terminals was already high enough to significantly alter actional potential dynamics. Work by the Parra lab in hippocampal slices suggests that changes in polarization due to tDCS can directly modify endogenous synaptic plasticity, by enhancing LTP and reducing LTD [22] [23]. It should however be noted that electric field strengths of $20 \mathrm{~V} / \mathrm{m}$ were used, which is 100 times stronger than the 0.2 
$\mathrm{V} / \mathrm{m}$ typically reached in the human head. Similar experiments with lower electric field strengths applied in in-vivo setups could shed more light on this.

While electric fields may improve task performance by increasing synaptic plasticity, it is also possible that they act by modifying the brain's network dynamics. Liu et al. postulate several mechanisms through which weak electric fields could induce spiking of neurons and alter network patterns [13]. A core mechanism may be stochastic resonance, in which low electric fields can bias the timing and probability of spikes in neurons that are nearing their spike threshold. This may then influence neural population coding that can ultimately cause cognitive and behavioral changes. There is evidence to support this theory, as electric fields as low as $0.2 \mathrm{~V} / \mathrm{m}$ have been shown to modulate firing rates periodically and affect network dynamics [24]. This would imply that tDCS has a modulatory impact on the neural state of the brain. The neural state, or brain state, can be described as reliable patterns of brain activity that involve the activation and/or connectivity of multiple large-scale brain networks [25]. The neural state is dynamic by nature, and alterations in this state will alter the brain's functioning in response to environmental demands [25]. If tDCS's effects are effectively caused by modulating the brain's neural state, inter-subject differences in baseline neural state could explain (some of) the substantial individual differences observed in tDCS learning paradigms. Likewise, this could explain the differential effectiveness of tDCS on different types of learning tasks, as distinct tasks may require specific neural states. Finally, it should be pointed out that the effect of the transcranial electric field on glial cells [26] and the immune system [27] is under investigation, but this is not further discussed in this article.

\section{$\underline{\text { tDCS transcutaneous mechanism }}$}

During tDCS, peripheral nerves in the scalp are exposed to electric fields of $20 \mathrm{~V} / \mathrm{m}$ and higher. These fields are strong enough to initiate action potentials in peripheral nerves which could then indirectly modify brain activity. Two nerves that are likely to be stimulated in conventional tDCS electrode montages are the greater occipital nerve and the trigeminal nerve (Figure 2). The greater occipital nerve arises from the $\mathrm{C} 2$ spinal nerve and innervates the posterior part of the scalp up to the vertex of the skull [28]. More anterior tDCS montages may stimulate the trigeminal nerve. This is the biggest cranial nerve whose nerve endings branch throughout the face, jaw, and forehead [29]. Interestingly, direct stimulation of the occipital and trigeminal nerves using pulsed stimulation are both recognized neuromodulation techniques with a long history [30]. The occipital and trigeminal nerves are interconnected [31] and process information in a number of parallel brain circuits. Afferent fibers from these nerves travel to the brainstem where they project to the nucleus of the solitary tract (NTS) and trigeminal nuclei [32] [33]. Next, information is integrated in the reticular formation: a large

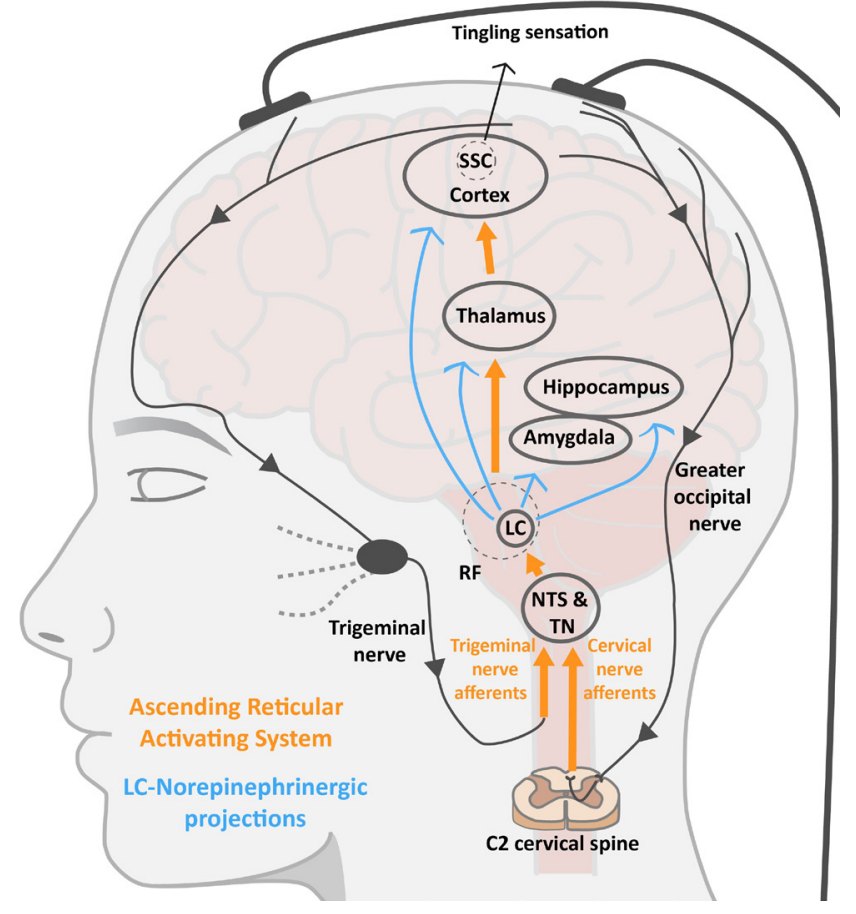

Figure 2. TDCS transcutaneous mechanism (LC = locus coeruleus, NTS = nucleus of the solitary tract, $\mathrm{RF}=$ reticular formation, $\mathrm{SSC}=$ somatosensory cortex, $\mathrm{TN}=$ trigeminal nuclei). 
network of nerves with nuclei clusters throughout the brainstem. Sensory information is then processed through the thalamus and somatosensory cortex, which mediates the tDCS tingling response. Importantly, the reticular formation participates in the ascending reticular activating system (ARAS): a system that integrates a wide variety of peripheral sensory information from cranial and spinal nerves. The ARAS contains a set of nuclei that release neurotransmitters in the cortex, both directly and through thalamic relays [34]. Through these nuclei, the ARAS exerts its influence on many basic behavioral processes, including arousal and vigilance [35]. One key nucleus in the ARAS is the locus coeruleus (LC), the main source of noradrenaline (NE) in the brain [33] [36]. Functional-imaging studies indicate that the LC-NE system is activated during situations that require attention, perceptual rivalry and memory retrieval [37]. We suggest that an increase in NE release mediated by LC activation [33] may be key to the learning effects of tDCS. More specifically, NE is known to increase cortical excitability [38], drive synaptic plasticity and modulate learning [39] and cognition [37]. Importantly, NE has already been reported as a mediator of tDCS effects [40] [41]. In these experiments, it was however not clear how tDCS drives NE release in the cortex. The transcutaneous mechanism provides a plausible answer, implicating the LC as the missing link. Direct input of the locus coeruleus to the hippocampus has moreover been shown to promote the formation of new memories through its noradrenergic [42] and dopaminergic [43] projections.

It is well characterized that many tDCS effects are polarity-specific, meaning that there is a different effect for anodal and cathodal tDCS stimulation on certain learning tasks for a specified electrode montage [44] [45]. Interestingly, suprathreshold stimulation of peripheral nerves is also polarityspecific with lower thresholds for cathodal stimulation [46] [47]. While we know that tDCS stimulates cranial nerves (as evidenced by the somatosensory response), we currently do not know if these effects are similar to those from a pulsed peripheral nerve stimulator. Recent research does however show that DC currents modulate peripheral nerves in a polarity-specific way [48]. Cathodal and anodal stimulation may thus stimulate the occipital and / or trigeminal nerve to a different degree, providing a putative explanation for tDCS's polarity specificity.

\section{Ambiguities in the tDCS Field}

The effects of tDCS on learning and memory in both humans and animals are very diverse. It seems unlikely that just one mechanism can account for all these effects. tDCS effects may be mediated by a range of different transcranial and potentially transcutaneous mechanisms, but this is currently unknown. Moreover, a recent surge in molecular research in the field is starting to shed light on the molecular mechanisms underpinning these effects [49] [50] [51]. Yet, it is unclear whether (and in what way) the molecular signature of cells is differentially altered by the transcranial and transcutaneous paths of tDCS. A current major issue in the tDCS field is that potential contributions from the transcutaneous route are not being controlled for nor investigated. In almost all tDCS experimental designs, a sham condition is used as control in which the tDCS is simply switched off. This control terminates both the transcranial and the putative transcutaneous tDCS routes, making it impossible to tell which of these routes is driving the observed tDCS effect.

\section{Improving experimental design}

The tDCS field could benefit from an improved experimental design that would allow for discrimination between transcranial and transcutaneous effects. To this aim, we suggest that new controls could be added to tDCS experiments. One such control could be 'transcranial-only' control groups, in which the 
transcutaneous route of tDCS is blocked. This means that all observed effects in this group can be attributed to the transcranial tDCS mechanism (i.e. the electric field in the brain). In human experiments, the transcutaneous route could be blocked by applying topical anesthetic creams on the scalp, under and around the electrodes. These anesthetics increase the threshold for firing action potentials by blocking sodium channels and stabilizing the membrane potential [52]. Consequently, peripheral nerve input will be blocked (or at least reduced) while leaving the electric field in the brain unchanged [10]. In animal in-vivo tDCS experiments, more invasive ways to evade the transcutaneous route may also be considered, such as implantation of electrodes directly on the skull or even sectioning of peripheral nerves. An alternative (or complementary) approach would be to implement 'transcutaneous-only' control groups in which the electric field in the brain is lacking. This can be achieved with active control groups targeting peripheral sites more distant to the brain, such as the neck, arms, and abdomen. In animals, transcutaneous-only stimulation could also be realized by directly implanting cuff electrodes around nerves. Finally, to see whether tDCS effects are brain-region specific, active controls can be applied to other positions on the scalp (note that this will only modify the transcranial and transcutaneous routes but not block any of them).

Standard use of these controls would help clarify to what extent tDCS effects are mediated by the transcutaneous or transcranial mechanism. Moreover, studies investigating the underlying mechanisms of tDCS could characterize how molecular and neurophysiological changes have a transcranial or transcutaneous basis. A clearer, improved understanding of the tDCS mechanism would give us the knowledge to direct research and resources towards novel tDCS approaches; improve effectiveness and reproducibility; and advance the field.

\section{References}

1 Salling, M.C. and Martinez, D. (2016) Brain Stimulation in Addiction. Neuropsychopharmacology 41, 2798-2809

2 Shiozawa, P. et al. (2014) Transcranial direct current stimulation for major depression: an updated systematic review and meta-analysis. Int. J. Neuropsychopharmacol. 17, 1443-1452

3 Berryhill, M.E. et al. (2014) Hits and misses: leveraging tDCS to advance cognitive research. Front. Psychol. 5, 800

4 Polanía, R. et al. Studying and modifying brain function with non-invasive brain stimulation. , Nature Neuroscience. (2018)

5 Rahman, A. et al. (2013) Cellular effects of acute direct current stimulation: Somatic and synaptic terminal effects. J. Physiol. 591, 2563-2578

6 Valero-Cabré, A. et al. Transcranial magnetic stimulation in basic and clinical neuroscience: A comprehensive review of fundamental principles and novel insights. , Neuroscience and Biobehavioral Reviews. (2017)

7 Tavakoli, A. V. and Yun, K. Transcranial alternating current stimulation (tACS) mechanisms and protocols. , Frontiers in Cellular Neuroscience. (2017)

8 Conde, V. et al. (2019) The non-transcranial TMS-evoked potential is an inherent source of ambiguity in TMS-EEG studies. Neuroimage DOI: 10.1016/j.neuroimage.2018.10.052

9 Siebner, H.R. et al. Distilling the essence of TMS-evoked EEG potentials (TEPs): A call for 
securing mechanistic specificity and experimental rigor. , Brain Stimulation. (2019)

Asamoah, B. et al. (2019) tACS motor system effects can be caused by transcutaneous stimulation of peripheral nerves. Nat. Commun. 10, 266

11 Chase, H.W. et al. (2020) Transcranial direct current stimulation: a roadmap for research, from mechanism of action to clinical implementation. Mol. Psychiatry 25, 397-407

Opitz, A. et al. (2015) Determinants of the electric field during transcranial direct current stimulation. Neuroimage 109, 140-150

Liu, A. et al. (2018) Immediate neurophysiological effects of transcranial electrical stimulation. Nat. Commun. 9,

14 Rampersad, S.M. et al. (2014) Simulating Transcranial Direct Current Stimulation With a Detailed Anisotropic Human Head Model. IEEE Trans. Neural Syst. Rehabil. Eng. 22, 441-452

So, P.P.M. et al. (2004) Peripheral nerve stimulation by gradient switching fields in magnetic resonance imaging. IEEE Trans. Biomed. Eng. DOI: 10.1109/TBME.2004.834251

Kessler, S.K. et al. (2012) Differences in the experience of active and sham transcranial direct current stimulation. Brain Stimul. DOI: 10.1016/j.brs.2011.02.007

17 Fanselow, E.E. (2012) Central mechanisms of cranial nerve stimulation for epilepsy. Surg. Neurol. Int. 3, S247-54

18 Mercante, B. et al. (2018) Anatomo-Physiologic Basis for Auricular Stimulation. Med. Acupunct. 30, 141-150

19 Radman, T. et al. (2009) Role of cortical cell type and morphology in subthreshold and suprathreshold uniform electric field stimulation in vitro. Brain Stimul. 2, 215-228.e3

20 Romero Lauro, L.J. et al. (2014) TDCS increases cortical excitability: Direct evidence from TMSEEG. Cortex 58, 99-111

21 Chakraborty, D. et al. (2018) Neuromodulation of axon terminals. Cereb. Cortex 28, 27862794

22 Kronberg, G. et al. (2017) Direct Current Stimulation Modulates LTP and LTD: Activity Dependence and Dendritic Effects. Brain Stimul. 10, 51-58

23 Kronberg, G. et al. (2019) Direct current stimulation boosts associative Hebbian synaptic plasticity and maintains its pathway specificity.

24 Reato, D. et al. (2010) Low-intensity electrical stimulation affects network dynamics by modulating population rate and spike timing. J. Neurosci. 30, 15067-15079

25 Tang, Y.Y. et al. (2012) Neural correlates of establishing, maintaining, and switching brain states. Trends Cogn. Sci. 16, 330-337

26 Gellner, A.-K. et al. (2016) Glia: A Neglected Player in Non-invasive Direct Current Brain Stimulation. Front. Cell. Neurosci. 10, 188

27 Hoare, J.I. et al. (2016) Electric fields are novel determinants of human macrophage functions. J. Leukoc. Biol. 99, 1141-1151

28 Cohen-Gadol, A. et al. (2011) The innervation of the scalp: A comprehensive review including anatomy, pathology, and neurosurgical correlates. Surg. Neurol. Int. 2, 178 

24pp. 1-15, KARGER

31 Busch, V. et al. (2006) Functional connectivity between trigeminal and occipital nerves revealed by occipital nerve blockade and nociceptive blink reflexes. Cephalalgia DOI: 10.1111/j.1468-2982.2005.00992.x

Monkhouse, S. (2005) Cranial nerves: Functional anatomy,

33 Tyler, W.J. et al. (2015) Transdermal neuromodulation of noradrenergic activity suppresses psychophysiological and biochemical stress responses in humans. Sci. Rep. 5, 1-17

34 Schwartz, M.D. and Kilduff, T.S. (2015) The Neurobiology of Sleep and Wakefulness. Psychiatr. Clin. North Am. 38, 615-644

35 Kinomura, S. et al. (1996) Activation by attention of the human reticular formation and thalamic intralaminar nuclei. Science (80-. ). DOI: 10.1126/science.271.5248.512

Couto, L.B. et al. (2006) Descriptive and functional neuroanatomy of locus coeruleusnoradrenaline-containing neurons involvement in bradykinin-induced antinociception on principal sensory trigeminal nucleus. J. Chem. Neuroanat. 32, 28-45

37 Sara, S.J. (2009) The locus coeruleus and noradrenergic modulation of cognition. Nat. Rev. Neurosci. 10, 211-223

38 Kuo, H.-I. et al. (2017) Acute and Chronic Noradrenergic Effects on Cortical Excitability in Healthy Humans. Int. J. Neuropsychopharmacol. 20, 634-643

Harley, C.W. (2004) Norepinephrine and dopamine as learning signals. Neural Plast. 11, 191204

40 Kuo, H.-I. et al. (2017) Acute and chronic effects of noradrenergic enhancement on transcranial direct current stimulation-induced neuroplasticity in humans. J. Physiol. 595, 1305-1314

41 Monai, H. et al. (2016) Calcium imaging reveals glial involvement in transcranial direct current stimulation-induced plasticity in mouse brain. Nat. Commun. 7,

42 Wagatsuma, A. et al. (2017) Locus coeruleus input to hippocampal CA3 drives single-trial learning of a novel context. Proc. Natl. Acad. Sci. U. S. A. 115, E310-E316

43 Kempadoo, K.A. et al. (2016) Dopamine release from the locus coeruleus to the dorsal hippocampus promotes spatial learning and memory. Proc. Natl. Acad. Sci. U. S. A. 113, 14835-14840

44 Reis, J. et al. (2009) Noninvasive cortical stimulation enhances motor skill acquisition over multiple days through an effect on consolidation. Proc. Natl. Acad. Sci. U. S. A. DOI: $10.1073 /$ pnas. 0805413106

45 Matsushita, R. et al. (2015) Polarity-specific transcranial direct current stimulation disrupts auditory pitch learning. Front. Neurosci. DOI: 10.3389/fnins.2015.00174

46 Hopp, F.A. et al. (1980) Effect of anodal blockade of myelinated fibers on vagal C-fiber afferents. Am. J. Physiol. Integr. Comp. Physiol. 239, R454-R462

47 Berger, J.J. et al. (1982) Electrode polarity and peripheral nerve stimulation. Anesthesiology $56,402-4$ 
and motor fibres in the human posterior tibial nerve, with a long-lasting effect on the H-reflex. Eur. J. Neurosci. 46, 2499-2506

49 Podda, M.V. et al. (2016) Anodal transcranial direct current stimulation boosts synaptic plasticity and memory in mice via epigenetic regulation of Bdnf expression. Sci. Rep. 6, 1-19

$50 \mathrm{Kim}$, M.S. et al. (2017) Repeated anodal transcranial direct current stimulation induces neural plasticity-associated gene expression in the rat cortex and hippocampus. Restor. Neurol. Neurosci. 35, 137-146

51 Yu, T.H. et al. (2019) Transcranial direct current stimulation induces hippocampal metaplasticity mediated by brain-derived neurotrophic factor. Neuropharmacology 144, 358367

52 Kumar, M. et al. (2015) Topical anesthesia. J. Anaesthesiol. Clin. Pharmacol. 31, 450 